\title{
Auditory event-related potentials associated with perceptual reversals of bistable pitch motion
}

\author{
Gray D. Davidson and Michael A. Pitts *
}

Department of Psychology, Reed College, Portland, OR, USA

\section{Edited by:}

Srikantan S. Nagarajan, University of California, San Francisco, USA

Reviewed by:

István Winkler, University of

Szeged, Hungary

Andrew R. Dykstra, University of

Heidelberg, Germany

\section{*Correspondence:}

Michael A. Pitts, Department of

Psychology, Reed College, 3203 SE

Woodstock Blvd., Portland, OR

97202, USA

e-mail:mpitts@reed.edu
Previous event-related potential (ERP) experiments have consistently identified two components associated with perceptual transitions of bistable visual stimuli, the "reversal negativity" (RN) and the "late positive complex" (LPC). The RN ( 200 ms post-stimulus, bilateral occipital-parietal distribution) is thought to reflect transitions between neural representations that form the moment-to-moment contents of conscious perception, while the LPC ( 400 ms, central-parietal) is considered an index of post-perceptual processing related to accessing and reporting one's percept. To explore the generality of these components across sensory modalities, the present experiment utilized a novel bistable auditory stimulus. Pairs of complex tones with ambiguous pitch relationships were presented sequentially while subjects reported whether they perceived the tone pairs as ascending or descending in pitch. ERPs elicited by the tones were compared according to whether perceived pitch motion changed direction or remained the same across successive trials. An auditory reversal negativity (aRN) component was evident at $\sim 170 \mathrm{~ms}$ post-stimulus over bilateral fronto-central scalp locations. An auditory LPC component (aLPC) was evident at subsequent latencies ( $350 \mathrm{~ms}$, fronto-central distribution). These two components may be auditory analogs of the visual RN and LPC, suggesting functionally equivalent but anatomically distinct processes in auditory vs. visual bistable perception.

Keywords: bistable perception, auditory awareness, ERP, consciousness

\section{INTRODUCTION}

Bistable stimuli refer to a class of physically unchanging stimuli that give rise to two mutually exclusive conscious percepts (Necker, 1832; Rubin, 1958; Bool et al., 1981; Lemmo, 2006). The use of bistable stimuli in functional neuroimaging and electrophysiological research allows one to correlate different brain states to different subjective percepts while holding sensory input constant. Neural correlates of bistable perception have been explored for a variety of static visual stimuli (e.g., Başar-Eroglu et al., 1993; Kornmeier and Bach, 2004; Pitts et al., 2007; see Sterzer et al., 2009 for a review), binocular rivalry (e.g., Lansing, 1964; Blake et al., 1992; Tong et al., 1998, 2006; Meng and Tong, 2004; Alpers et al., 2005; Pitts and Britz, 2011), apparent motion stimuli (Sperling et al., 1985; Müller et al., 1999; Kaneoke et al., 2009; Kim et al., 2009; Genç et al., 2011; Strüber et al., 2014), and more recently, auditory streaming stimuli (Gutschalk et al., 2005; Dykstra et al., 2011; Kashino and Kondo, 2012; see Snyder et al., 2012 for a review).

In order to time-lock electrophysiological recordings to stimulus onset, a number of studies have employed intermittent (as opposed to constant) stimulus presentation methods (Leopold et al., 2002; Kornmeier and Bach, 2004, 2005, 2006, 2009, 2012; Kornmeier et al., 2007, 2009, 2014; Pitts et al., 2007, 2008; Britz et al., 2009; Intaite et al., 2010, 2013, 2014; Ehm et al., 2011; Pitts and Britz, 2011). Intermittent paradigms, particularly those in which each trial consists of a brief stimulus (e.g., $\sim 800$ ms stimulus duration) followed by a brief blank interval (e.g., $\sim 400 \mathrm{~ms}$ inter-stimulus interval (ISI)), have been shown to constrain perceptual reversals to occur only at stimulus-onset while approximating reversal rates found in constant presentation paradigms, i.e., reversals occur every $\sim 2-6 \mathrm{~s}$ (Orbach et al., 1963, 1966; Leopold et al., 2002; Britz et al., 2009; Kornmeier et al., 2009). Subjects are trained to report when a perceptual reversal occurs across adjacent trials or to report their specific percept after each individual trial. In either case, brain activity elicited by each stimulus-onset can be sorted according to whether perception reversed or remained stable relative to the previous trial.

Previous studies that compared event-related potentials (ERPs) for reversal vs. stable trials have consistently reported two components: the "reversal negativity" (RN), a negative-going difference for reversal vs. stable trials over bilateral occipitalparietal scalp regions from $\sim 170-350 \mathrm{~ms}$; and the "late positive complex" (LPC), a positive-going difference between reversal and stable trials over the central-parietal scalp from $\sim 350-600 \mathrm{~ms}$ (Başar-Eroglu et al., 1993; Kornmeier and Bach, 2004; Pitts et al., 2007; Britz et al., 2009; Koivisto and Revonsuo, 2010). Based on the estimated locations of their neural generators as well as their timing and sensitivity to top-down and task-based manipulations, it has been suggested that the $\mathrm{RN}$ is a neural marker of the transition between the two perceptual representations (but see 
Intaite et al., 2010), while the LPC reflects the outcome of this perceptual change, e.g., the updating of working memory required to perform the perceptual-reporting task (Pitts et al., 2008, 2009; Pitts and Britz, 2011).

While a number of experiments have replicated the $\mathrm{RN}$ and LPC effects using a variety of stimuli and presentation parameters (e.g., Kornmeier and Bach, 2004; Pitts et al., 2007; Britz et al., 2009; Intaitè et al., 2010), analogous reversal-related components have not yet been identified in sensory modalities other than vision. The most well developed bistable auditory paradigm to date consists of a melodic ABA sequence that can be perceived as two types of continuous patterns: a repeating lo-hi-lo triplet, or two continuous streams at different frequencies (Bregman, 1994; Gutschalk et al., 2005; van Noorden, unpublished). Pressnitzer and Hupé (2006), using the ABA auditory stimulus and a matched visual stimulus, found equivalent distributions of perceptual durations across modalities (short percept durations were common and the frequency of longer stable periods decreased monotonically), while the duration of a given stable period was not correlated to the duration of the previous stable period (suggesting that reversals in both modalities were stochastic). Furthermore, when subjects were instructed to voluntarily maintain a given percept as long as possible, percepts inevitably switched on a comparable number of trials across modalities. Taken together, these findings suggest the existence of auditory equivalents to visually bistable figures, at least in terms of perceptual dynamics (Pressnitzer and Hupé, 2006).

The neural basis of auditory bistability has been explored in a limited number of studies (see Gutschalk and Dykstra, 2014 for a review). Although a wide range of methods and stimuli have been employed (e.g., Sato et al., 2004; Cusack, 2005; Gutschalk et al., 2005; Snyder et al., 2006; Kondo and Kashino, 2007, 2009; Snyder et al., 2009; Dykstra et al., 2011; Schadwinkel and Gutschalk, 2011; Kondo et al., 2012), to our knowledge no previous study has combined the intermittent paradigm with ERPs in the auditory domain. In order to obtain ERPs in an intermittent bistable auditory paradigm comparable to visual paradigms, a novel stimulus is required as the streaming ABA stimulus is not directly amenable to this approach. To create such a stimulus, we began by exploring stimuli with ambiguous pitch characteristics, namely "tritone" stimuli which are derived from Shepard tones.

Shepard tones (Shepard, 1964, 1982) are complex auditory stimuli possessing pitch class information but lacking pitch height information (e.g., a Shepard tone might be recognizable as pitch class C\# while the specific C\# octave is ambiguous). These tones are typically composed of ten octave-related pure-tone harmonics, the amplitudes of which are constrained by a Gaussian envelope as shown in Figure 1. When subjects are asked to make judgments about the direction of pitch motion between paired Shepard tones they inevitably use proximity as the key factor. Thus, a pair of Shepard tones built respectively on pitch classes $\mathrm{C \#}$ and $\mathrm{D}$ will be perceived as ascending in pitch (a distance of 1 semitone) rather than descending (a distance of 11 semitones). When a pair of Shepard tones are exactly six semitones apart, an interval called a tritone, listeners can no longer rely on proximity to make judgments about pitch motion but will still confidently report perceiving either an ascending or descending pitch motion.

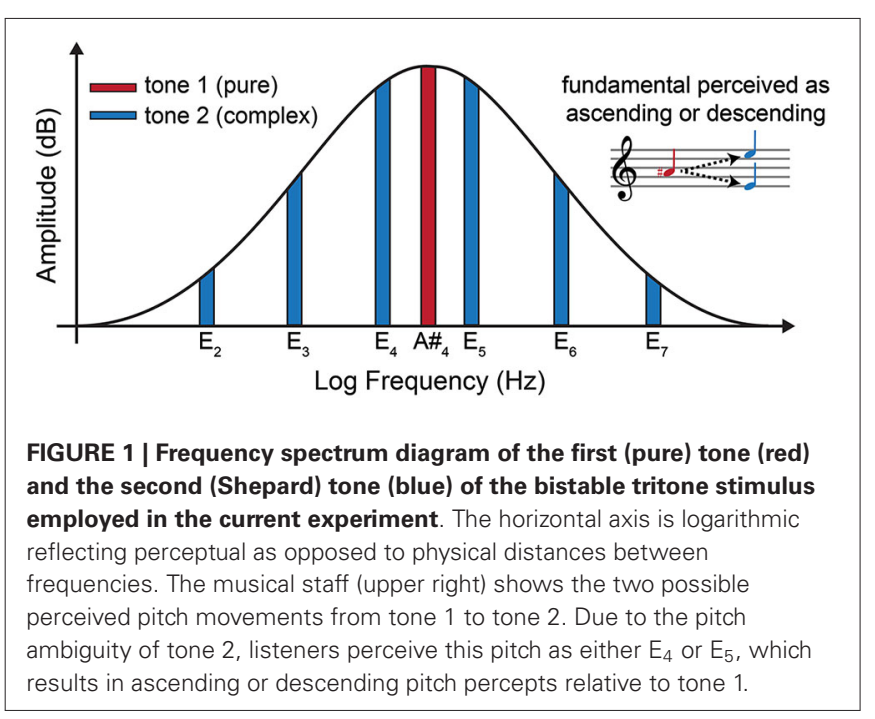

Moreover, transposition up or down the scale (while maintaining the ambiguous six semitone relationship) leads the same listeners to reverse their pitch motion judgments and different listeners will often hear opposite movements when listening to the same tone pair (Deutsch, 1986). These effects are collectively referred to as the "tritone paradox" and have been extensively investigated by Diana Deutsch et al. (Deutsch, 1987, 1991, 1992, 1994, 1997; Deutsch et al., 1987; Ragozzine and Deutsch, 1994; Repp, 1994, 1997; Giangrande, 1998; Chalikia and Leinfelt, 2000; Chalikia et al., 2000).

Previous investigations of the tritone paradox suggest that each individual possesses a peak pitch class, which represents the turning point at which their perception will reverse between ascending and descending pitch motion as the stimulus is transposed around the pitch class circle (Deutsch et al., 1987; Deutsch, 1991, 1994; Ragozzine and Deutsch, 1994; Chalikia and Leinfelt, 2000; Chalikia et al., 2000). For tone pairs starting on this peak pitch class, subjects report nearly equal ascending and descending perceptions across trials. For tone pairs away from the peak pitch class, subjects show a strong bias to perceive a particular pitch motion, but rarely $100 \%$, meaning that for nearly all listeners, any pair of tritone stimuli can be perceived as bistable (Deutsch, 1986, 1987). Indeed preliminary tests showed that with minimal training, most listeners can be taught to hear paired Shepard tones as ascending and descending with equal probability. Tritone stimuli therefore, may be ideally suited to serve as bistable auditory stimuli. Surprisingly, only a few previous studies have noted the bistable characteristics of Shepard tone pairs (Giangrande et al., 2003; Repp and Knoblich, 2007; Repp and Thompson, 2010).

The current investigation utilized a variant of Deutsch's tritone stimuli to induce bistable perception in the intermittent paradigm. Pairs of tones were constructed such that the first tone was unambiguous (a pure tone) and the second tone was a Shepard tone, six semitones apart from the first (i.e., a tritone). Thus, on any given trial, subjects perceived the pitch motion between the two tones as ascending or descending while the physical properties of the tones remained constant. Behaviorally, we sought to determine whether this particular auditory bistable stimulus exhibits 
characteristics common for visual bistable figures, e.g., mutual exclusivity, inevitability of reversals, unpredictability of reversals, (Leopold and Logothetis, 1999). We also recorded the EEG, timelocked ERPs to the onset of each tone pair, and compared ERPs according to whether perceived pitch direction changed (reversal) or stayed the same (stable) across adjacent trials. These ERP comparisons allowed us to identify potential auditory analogs to the visual RN and LPC components.

\section{METHODS \\ PARTICIPANTS}

Twenty-six Reed College students and recent alumni (ages 18-30, $52 \%$ female) participated as volunteers. In exchange for participation, subjects were entered into a lottery with a chance to win $\$ 150$. Written informed consent was obtained from each subject prior to participation in the experiment. No participant reported a history of brain injury or any other neurological condition that might affect his or her electrical brain activity. All procedures adhered to federal regulations and were approved by the Reed College Institutional Review Board. After analysis, five subjects were excluded due to insufficient numbers of trials in at least one condition (less than 100). The final analysis included the remaining 21 participants.

\section{STIMULI}

Each stimulus consisted of a pair of tones: a pure tone at 466.164 Hz. (an A\#) followed by a complex Shepard tone built from six octave-related sinusoidal harmonics, each in pitch class $\mathrm{E}$ (pitches related perceptually by octaves doubled in frequency: 82.407 Hz, $164.814 \mathrm{~Hz}, 329.628 \mathrm{~Hz}, 659.256 \mathrm{~Hz}, 1318.512 \mathrm{~Hz}$, and $2637.024 \mathrm{~Hz}$ ). These particular tones were selected because the experiment was to be conducted on the west coast of the United States, and Shepard tone pairs built on A\# and E have been shown to be most commonly ambiguous for Californian listeners (Deutsch, 1991). Each tone was presented for $400 \mathrm{~ms}$ in immediate succession (no silent interval between tones). Each tone pair was separated by a silent ISI lasting a random duration between 500-900 ms. During each block of trials, a sequence of 65 tone pairs was presented. A spectral diagram of the first and second tones is provided in Figure 1. Details concerning the construction of these tones can be found in Deutsch (1987). The tone pairs employed in the current study differed from Deutsch's tritone paradox (Deutsch, 1986, 1987) in that the first tone was a pure tone rather than a Shepard tone. This modification confines pitch ambiguity to the second tone, thus allowing ERP analyzes to focus on neural responses to the onset of the second tone. All stimuli were presented at $\sim 80 \mathrm{~dB}$ via two Logitech Surround Sound Speakers (model Z906; frequency response: $35 \mathrm{~Hz}-20$ $\mathrm{kHz})$, positioned at equal distances $(1.5 \mathrm{~m})$ from the participant's ears.

\section{PROCEDURE}

All experiments were conducted in a sound-attenuated, electrically shielded booth (Industrial Acoustics Company). While the EEG cap was being prepared and electrical impedances lowered, subjects practiced perceiving the stimuli until they reported fluency of judgment between ascending and descending perceptions.
Because previous studies have found that reversal rates increase during the initial trials of an experiment with novel bistable stimuli (Long and Toppino, 2004), each participant was exposed to a minimum of two blocks of stimuli (130 total) before EEG recording began.

Participants maintained fixation on a small, centrally located fixation cross (subtended angle of $0.76^{\circ}$ ) which was visible throughout all stimulus presentations and ISIs. Subjects were trained to report their perception after each stimulus pair by pressing one of two buttons with the index or middle finger of the right hand, indicating ascending or descending pitch perception (buttons counterbalanced across participants). In the case that a tone pair sounded ambiguous with respect to direction of pitch motion, participants were instructed to refrain from pressing any buttons. The time window for viable responses was set to 0-1300 ms after tone 1 onset and any trials in which subjects' responses fell outside this window were excluded.

Trials were presented in blocks of 65 , separated by short rest breaks of approximately $15 \mathrm{~s}$. Sets of four blocks were separated by longer 2-min breaks, and each subject completed 12 or 16 blocks for a total of 780 or 1040 total trials. Trials were segregated into conditions by comparing the reported perception on a given trial to the perception on the previous trial. A trial was considered a reversal if reported percepts differed between adjacent trials and stable if perception remained the same. The experiment commenced after 12 blocks ( 3 sets of 4 ) if each of these two conditions (reversal and stable) contained at least 150 trials; otherwise an additional set of four blocks was administered. Figure 2 shows a schematic of the stimulus presentation sequence and perceptual reporting task.

\section{EEG/ERP METHODS}

EEG scalp voltages were recorded using a 96-channel electrode cap with equidistant electrode placements (EasyCap). Signals were amplified via $3 \times 32$ BrainAmp Standard amplifiers (Brain Products), bandpass filtered between 0.1 and $150 \mathrm{~Hz}$, and digitized at $500 \mathrm{~Hz}$. During data collection all channels were referenced to $\mathrm{CPZ}$ and were re-referenced to the average of the left and right mastoids offline. Eye position and eye movements were monitored via a vertical electrooculogram (EOG) channel positioned under the left eye and left and right horizontal EOG channels (re-referenced to each other offline to form a bipolar pair). All sensors were individually adjusted until the impedance of each was less than $5 \mathrm{k} \Omega$.

ERPs were time-locked to the onset of the first stimulus, and separated into reversal and stable conditions as described above. Trials with eye movement, blink, or muscle artifacts within a time window $-100 \mathrm{~ms}$ to $+1000 \mathrm{~ms}$ (relative to tone 1 ) were detected and rejected semi-automatically by a combination of computerbased peak-to-peak amplitude thresholds and visual inspection. Trials in each condition were averaged, low-pass filtered at $30 \mathrm{~Hz}$, and baseline corrected from -100 to $0 \mathrm{~ms}$ prior to tone 1 onset. Because pitch ambiguity occurred only for the second tone, the timing of all ERP effects are reported with respect to the onset of tone 2 (hereafter, time-zero = tone-2-onset).

Repeated measures analysis of variance (ANOVA) were carried out on mean amplitudes within latency windows corresponding 


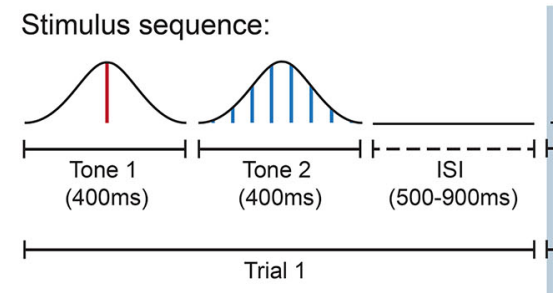

Example percepts:

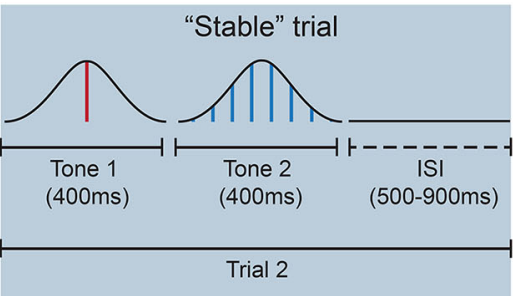

Trial 2

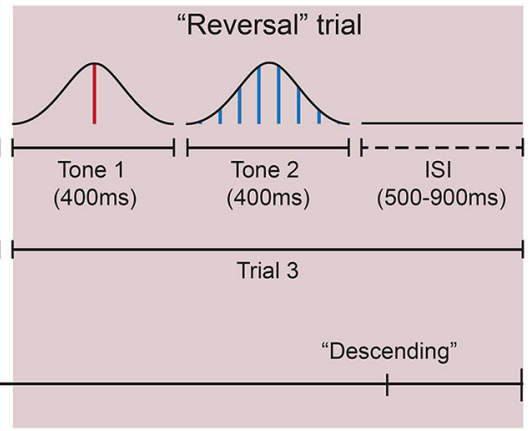

FIGURE 2 | Intermittent stimulus presentation sequence and corresponding example percepts across trials. In this example, the subject reports the perceived pitch direction of the tone pair as ascending in the first trial (white background) and also ascending in the second trial (blue background). Thus the second trial would be categorized as a "stable" trial because perceived pitch direction remained the same across successive trials. The
"Ascending" tone pair in the third trial (red background) was perceived as descending and would be categorized as a reversal trial because perceived pitch direction differed from the immediately preceding trial. Each tone was presented for $400 \mathrm{~ms}$ in immediate succession, while trials were separated by a silent ISI of variable duration (500-900 ms). Subjects indicated their perceived pitch direction after each tone pair. to amplitude differences observed in the grand average ERPs for the reversal and stable conditions. An auditory reversal negativity (aRN) was apparent from 120-220 ms, bilaterally over frontocentral electrode sites 9, 10, 22, 23, 24, 38, 39, 21, 37 and 17, $18,32,33,34,51,52,35,53$ (corresponding to electrodes C4, C6, FC4, FC6, F2, F4, F6, AF4 and C3, C5, FC3, FC5, F1, F3, F5, and AF3 of the International 10/20 system), and was assessed via $2 \times 2 \times 9$ ANOVA with the factors perception (reversal vs. stable), hemisphere (left vs. right), and electrode (channels listed above). An auditory late positive complex (aLPC) was evident at subsequent latencies (320-380 ms) at electrodes 2, 3, 6, 7, 8, 19, 20 (corresponding to C1, C2, FCZ, F1, FZ, F2 and AFZ), and was assessed by a $2 \times 7$ ANOVA with the factors perception and electrode. Exploratory analyzes were conducted in a third ANOVA on a difference component that was evident just prior to the onset of tone 2 , from -80 to $-20 \mathrm{~ms}$, over fronto-central electrodes 2, 7, 8, 19, and 20 (corresponding to FCZ, F1, FZ, F2 and AFZ).

\section{RESULTS \\ BEHAVIORAL RESULTS}

Overall, participants reported perceiving ascending pitch motion on $51.2 \%$ of all trials, descending pitch motion on $45.84 \%$ of trials, and ambiguous or unclear percepts on $2.96 \%$ of trials. These results suggest mutual exclusivity of percepts elicited by this bistable stimulus. Individual perceptual biases varied in both directions, with one subject reporting ascending vs. descending pitch on $39 \%$ and $61 \%$ of trials, respectively, and another reporting the same percepts with an opposite bias: $60 \%$ ascending and $40 \%$ descending.

Perceptual reversals of pitch motion occurred on average every 2.92 trials $(4.38 \mathrm{~s})$, and followed a monotonically decreasing distribution (Figure 3), meaning the probability of a stable period continuing decreased steadily on each trial. An exponential decay function fitted to these data points matched closely $\left(r^{2}=0.98\right)$. The shape of this curve indicates that reversals were inevitable since the probability that a reversal will not occur decreased asymptotically to zero. Reversals were also shown to be unpredictable, meaning that a given reversal could not be predicted by perceptual reports on preceding trials. In particular, the correlation between the lengths of adjacent stable periods (as measured by number of stable perceptions before a reversal) was low $(r=$ 0.147 ). Mean reaction time, collapsed across both conditions was $838.6 \mathrm{~ms}$ post-tone-2-onset ( $\mathrm{sd}=150.0$ ), and did not differ statistically between ascending and descending percepts $(p=0.945)$ or between stable and reversal trials $(p=0.701)$.

\section{ERP RESULTS}

For ERP analyses, the overall percentage of trials in each condition was $65.42 \%$ stable and $34.58 \%$ reversal. Minimum trials per subject for each condition were 186 stable and 102 reversal. Mean number of trials per condition across subjects was $389(\mathrm{sd}=153)$ stable and $201(\mathrm{sd}=84)$ reversal.

A negative-going amplitude difference for reversal trials (aRN) was evident from 120-220 ms (post-tone-2-onset) over frontocentral scalp locations, $F_{(1,20)}=15.2, p<0.001$, partial etasquared $=0.43$ (mean amplitudes: reversal $=-2.8 \mu \mathrm{V}$ (sem $=$ $0.53)$; stable $=-2.0 \mu \mathrm{V}($ sem $=0.58))$. This component was present in both hemispheres with a clear bilateral distribution. The hemisphere $\times$ condition interaction was not significant, $F_{(1,20)}=0.017, p=0.9$. No other effects or interactions approached significance.

A subsequent, positive-going difference for reversal trials (aLPC) was apparent from 320-380 ms (post-tone-2-onset) with a midline distribution over the fronto-central scalp, $F_{(1,20)}=6.2$, $p=0.02$, partial eta-squared $=0.24$ (mean amplitudes: reversal $=$ $-6.0 \mu \mathrm{V}(\mathrm{sem}=0.62)$; stable $=-6.9 \mu \mathrm{V}(\mathrm{sem}=0.71))$. ERPs from the reversal and stable conditions at electrode sites centered on the aRN and aLPC components are shown in Figure 4. Difference waves were computed by subtracting stable ERPs from reversal ERPs. Scalp topographies of these difference waves are provided in Figure 5. 


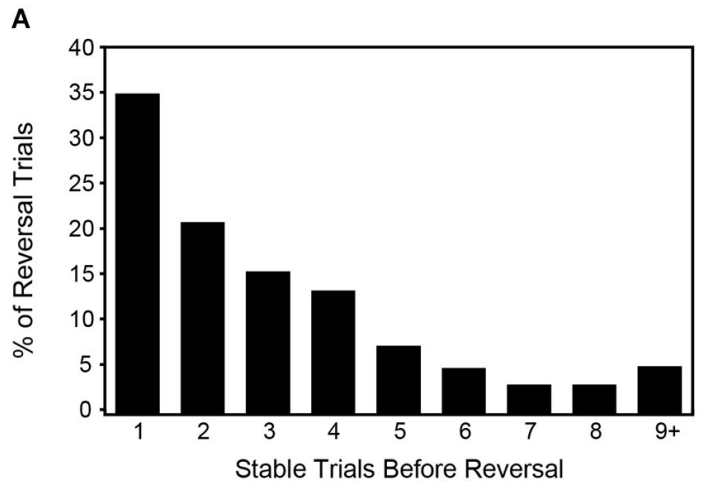

FIGURE 3 | Behavioral data showing the number of stable trials preceding a reversal trial as a function of the overall frequency of reversal trials (A), and the cumulative probability of a reversal occurring
B

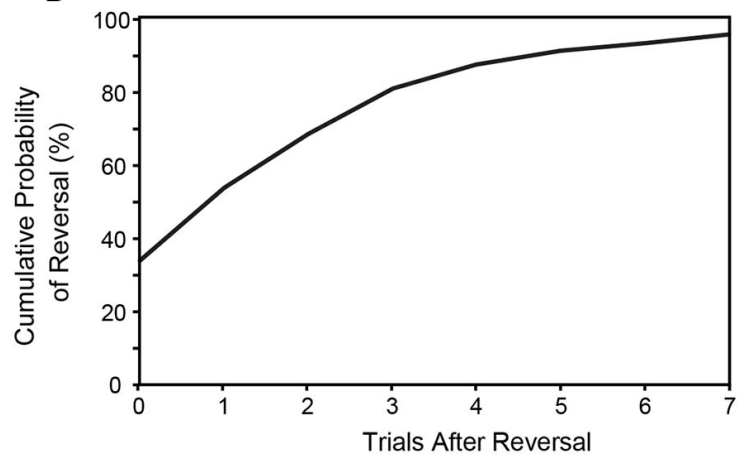

as a function of trials following a reversal (B). Both figures demonstrate the inevitability of perceptual reversals over time, a key feature of bistable stimuli.

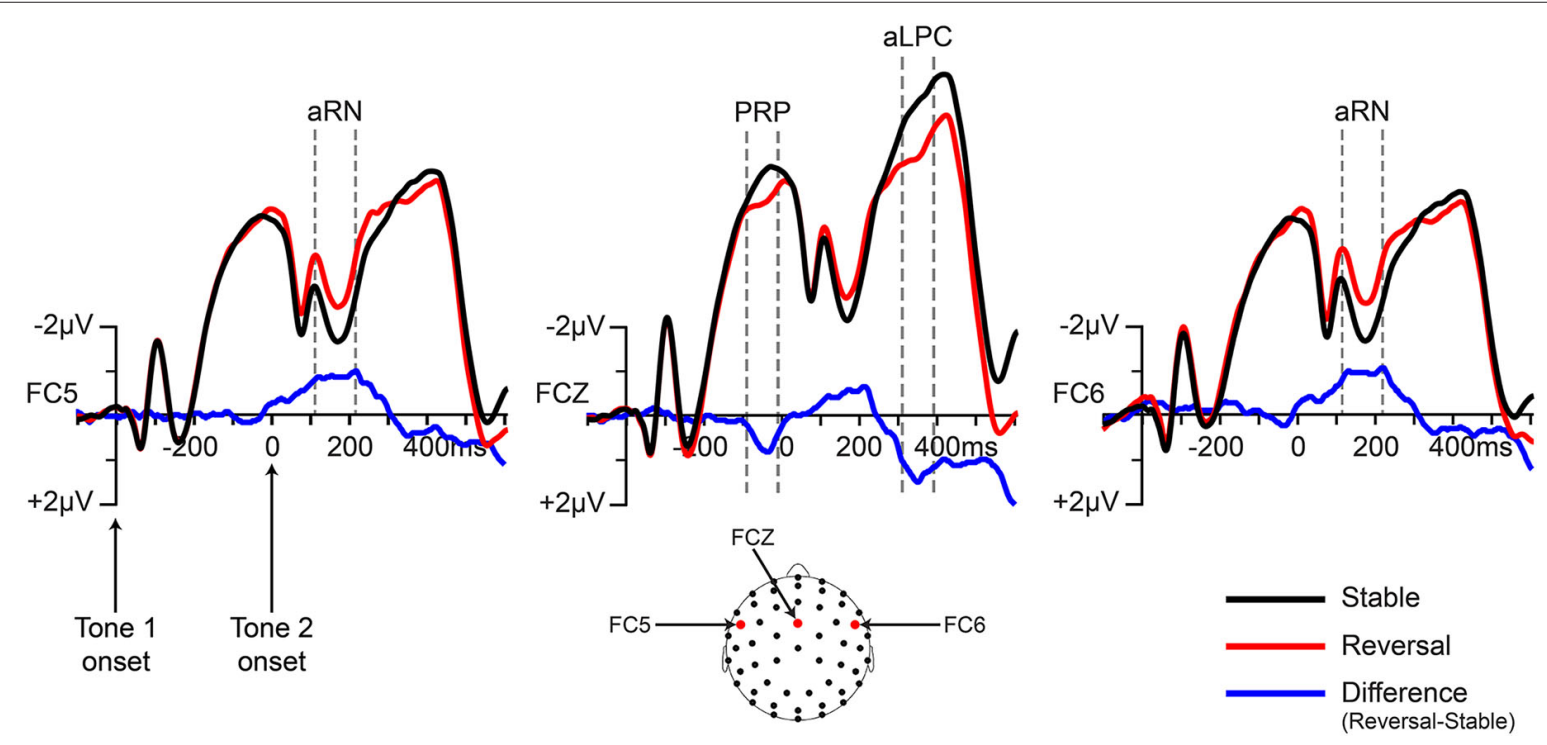

FIGURE 4 | Grand-averaged ERPs for stable and reversal trials and difference waves (reversal minus stable) at electrodes representative of the observed amplitude differences. ERPs were time-locked to tone 1, but note that the time scale is adjusted such that tone-2-onset was treated as time zero for analysis purposes (because tone 2 was the ambiguous tone). The time windows of the main components of interest are denoted by the dotted gray lines (see main text for details): aRN = auditory reversal negativity; aLPC = auditory late positive complex; PRP = pre-reversal positivity.
An unexpected ERP difference was observed during a time window immediately prior to the onset of tone $2(-80$ to $-20 \mathrm{~ms}$ ). The paired presentation sequence, with an unambiguous tone followed by an ambiguous tone, allowed for investigation of brain activity preceding perceptual reversals, i.e., during the time window between tone-1-onset and tone-2-onset. The prereversal ERP difference observed here had a very similar scalp distribution to the aLPC and was tested over similar electrode sites. This difference trended towards statistical significance, $F_{(1,20)}=$ 2.93, $p=0.1$, partial eta-squared $=0.13$ (mean amplitudes: reversal $=-4.9 \mu \mathrm{V}(\mathrm{sem}=0.51)$; stable $=-5.4 \mu \mathrm{V}(\mathrm{sem}=0.57))$, and should be investigated in future experiments that utilize paired bistable stimuli. We refer to this difference component here as the pre-reversal positivity (PRP).
Finally, we compared ERPs elicited by the bistable tritone stimuli according to whether subjects perceived ascending vs. descending pitch motion (i.e., percept A vs. B instead of reversal vs. stable). This comparison revealed no amplitude differences at any time point, for any electrode on the scalp. While it is reasonable to assume that there must be some difference in neural activity underlying the two perceptual states, scalp ERP measures were unable to resolve this difference.

\section{DISCUSSION}

The goals of this study were to create a bistable auditory stimulus suitable for use with the intermittent ERP paradigm, test the perceptual dynamics of this stimulus, and identify potential auditory analogs of the RN and LPC components commonly found 


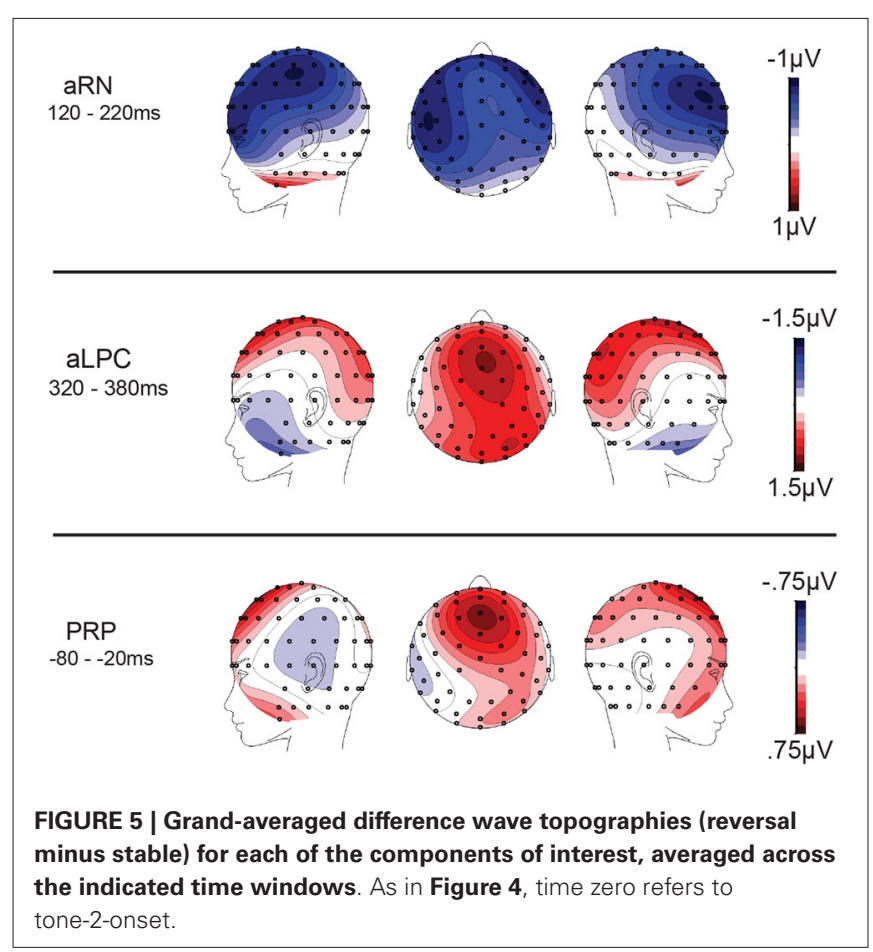

for visual bistable figures. The physical features of the tritone stimulus remained constant throughout the experiment while subjects reported their alternating perceptions of pitch motion (ascending or descending pitch) after each trial. Behaviorally, we found that this auditory bistable stimulus shared similar qualities to visual bistable figures, including mutual exclusivity of the two alternative percepts, inevitability of perceptual reversals over time, and unpredictability of reversals for any given trial. The ERP results revealed two difference components at similar (slightly earlier) latencies as the visual RN and LPC, but with more frontal (compared to posterior) scalp distributions. We provisionally refer to these two difference components as the aRN and aLPC.

\section{PERCEPTUAL DYNAMICS OF THE BISTABLE TRITONE STIMULUS}

Our behavioral results showed that for this particular derivation of the tritone stimulus, reversals typically occurred after a small number of trials (every $\sim 3$ trials or $\sim 4.5 \mathrm{~s}$ ). We further found that reversal intervals decreased monotonically, indicating that on a long enough timescale the probability that a stable period will continue decreases to zero and therefore reversals are inevitable. The two alternative percepts were reported with near equal probability ( $51 \%$ vs. $46 \%$ ) while ambiguous or otherwise unclear percepts were only reported on $3 \%$ of trials. This finding confirms the mutual exclusivity of percepts elicited by the tritone stimulus. Finally, on any given trial, reversals were found to be nearly stochastic, with a very low correlation between the lengths of adjacent stable periods, and thus very low predictability for the timing of reversals. Randomness of reversals is a property commonly attributed to visual bistable figures. Overall, these results suggest that the perceptual dynamics of the tritone stimulus employed here are similar to those found for most visual bistable stimuli (Leopold and Logothetis, 1999; Leopold et al., 2002; Long and Toppino, 2004; Pressnitzer and Hupé, 2006; Sterzer et al., 2009).

\section{AUDITORY VS. VISUAL ERP COMPONENTS ASSOCIATED WITH PERCEPTUAL REVERSALS}

In the visual domain, two components have been widely reported for perceptual reversals of bistable figures, the RN and LPC. In the current study, potential auditory counterparts to the visual RN and LPC components were identified. The aRN, reported in this experiment was elicited earlier than the visual RN: $120-220 \mathrm{~ms}$ post-tone-2 in the current study, relative to $\sim 180-280 \mathrm{~ms}$ in previous visual studies. The latency of the aLPC was similarly reduced (320-380 ms post-tone-2) compared to the visual LPC (350$600 \mathrm{~ms}$ ). Reduced latencies are generally expected for auditory vs. visual ERPs, as the earliest scalp ERP in the auditory modality, the P1 at 20-50 ms, precedes the earliest visual ERP, the $\mathrm{C} 1$ at 50-100 ms (Davis, 1939; Spehlmann, 1965; Hillyard et al., 1973; Näätänen and Picton, 1987; Clark et al., 1994).

In addition to latency differences between modalities, the aRN and aLPC showed more frontally-focused scalp distributions compared to the visual RN and LPC reported previously. The aRN was strongest bilaterally at fronto-central locations while the visual $\mathrm{RN}$ is typically restricted to parietal and occipital sites (Pitts et al., 2007, 2008; but see Intaitè et al., 2010; Kornmeier and Bach, 2012). Similar to the visual RN, the aRN temporally overlaps the N1/P2 but does not appear to reflect a modulation of the $\mathrm{N} 1$ or $\mathrm{P} 2$ peaks as evidenced by marked differences in scalp topography, i.e., the N1 and P2 peaks showed a broad central or fronto-central distribution centered on the midline, while the aRN was clearly bilateral. The aLPC was centered on the midline at fronto-central electrode sites compared to the visual LPC which, although widespread, generally centers on parietal and parieto-central electrodes (Kornmeier and Bach, 2004; Pitts et al., 2008, 2009). It will be worthwhile for future studies to estimate the locations of the neuroanatomical sources of the aRN and aLPC, as both components can be isolated via difference waves while the physical stimulus remains identical across the two conditions.

It has previously been suggested that the visual RN reflects the process of updating the contents of consciousness in higherlevel (ventral stream) visual areas (Pitts and Britz, 2011). The existence of a reversal ERP component in a second modality, sharing similar timing and polarity, while differing in ways which are expected due to characteristic differences between the two perceptual systems, supports the view that the visual RN and aRN reflect common perceptual processes across modalities. However, the current study as well as previous visual studies, have not yet conclusively demonstrated a link between the RN component and the transition between contents of conscious perception.

Intaite et al. (2010) argue that the $\mathrm{RN}$ does not reflect changes in conscious perception because in one of their conditions, exogenous reversals of unambiguous Necker cubes did not elicit an RN. It is justifiably assumed that any correlate of conscious perceptual change found for endogenous reversals of ambiguous 
stimuli should also be apparent for exogenous reversals of unambiguous stimulus variants. Kornmeier and Bach (2004), however, reported an RN component for exogenous reversals of unambiguous Necker cubes, and interestingly this exogenous RN was found to occur at earlier latencies compared to the endogenous RN. An interesting extension of the current experiment would be to measure ERPs elicited by both ambiguous tritone stimuli and unambiguous variants in which reversals in pitch perception are controlled exogenously. If the aRN, like the visual RN, indeed reflects changes in conscious content, it should also be found for exogenous reversals of pitch motion, perhaps at slightly earlier latencies.

\section{RELATIONSHIP BETWEEN THE aRN AND OTHER AUDITORY ERP COMPONENTS}

An alternative interpretation of the visual and auditory RN components is that they both reflect shifts in attention that immediately precede or follow the establishment of new perceptual content. Intaitè et al. (2010) employed bilateral displays to test whether the $\mathrm{RN}$ is simply an N2pc component in disguise. The $\mathrm{N} 2 \mathrm{pc}$ is a well-studied ERP component that reflects shifts in visual spatial attention (Luck and Hillyard, 1994; Hickey et al., 2006; Robitaille and Jolicoeur, 2006; Luck, 2012). An auditory equivalent to the $\mathrm{N} 2 \mathrm{pc}$, labeled the $\mathrm{N} 2 \mathrm{ac}$, has recently been discovered and is thought to reflect similar attention shifts in the auditory domain (Gamble and Luck, 2011). Intaite et al. (2010), however, found that the RN was distinct from the N2pc component suggesting that the $\mathrm{RN}$ reflects some other process besides the shifting of visual spatial attention. One hypothesis is that a transition in object-based attention is a necessary prerequisite for perceptual reversals and that the $\mathrm{RN}$ reflects this non-spatial type of attention shift. Alternatively, perceptual reversals might attract object-based attentional resources. In this case, the attention shift (and the RN) would be considered a consequence of conscious perceptual change. Currently, the relationship between attention and conscious perception is an open question and a topic of intense debate (Tsuchiya and van Boxtel, 2013). It will be important for future studies to try to tease apart whether the RN and aRN are more closely associated with transitions in conscious perceptual content or with preceding/consequential attentional shifts.

A separate concern with the current $\mathrm{aRN}$ results is that this ERP difference component might reflect auditory-change detection more generally. The mismatch negativity (MMN), a wellknown auditory ERP component with similar timing, polarity, and scalp distribution as the aRN, is typically elicited by deviant (less probable) stimuli within a sequence of standard (more probable) stimuli (Näätänen et al., 1978, 2004). In the present experiment, participants perceived the tritone stimuli as ascending or descending in pitch with near equal probability (51.2\% Ascending vs. $45.8 \%$ Descending), but perceptual reversals of pitch motion were experienced far less often than perceptual stability $(65.42 \%$ stable vs. $34.58 \%$ reversal), thus rendering reversal trials as a perceptual "deviant" and stable trials as the "standard". Because reversal rates varied across subjects, we were able to test whether the aRN and the MMN are indeed the same component by comparing individual subject aRN amplitudes with individual reversal rates. It has commonly been found that decreasing the probability of the deviant stimulus increases the amplitude of the MMN (Näätänen and Kreegipuu, 2012). Thus, if the aRN is identical to the MNN, larger amplitudes would be expected for subjects in which reversals were rarer and smaller amplitudes for subjects in which reversal and stable trials were more equivalent. This analysis, however, did not reveal even a modest relationship between aRN amplitude and reversal probability $(r=0.094, p=$ 0.69 ), suggesting that the aRN and MMN are most likely distinct components.

A third component that the aRN might relate to is the awareness related negativity (ARN), reported in previous auditory masking experiments (Gutschalk et al., 2008; Königs and Gutschalk, 2012; Wiegand and Gutschalk, 2012). The ARN, a negative-going component from $\sim 100-250 \mathrm{~ms}$, has been found to uniquely index detected tones (compared to undetected, physically identical tones) when the target tones are presented within a complex multi-tone background (Gutschalk and Dykstra, 2014). While Gutschalk and colleague's methods did not include intermittent bistable stimuli, nor did the present investigation make aware vs. unaware comparisons, it is possible that changes in auditory awareness is the common underlying factor across these studies. A visual analog of the ARN, the visual awareness negativity (VAN), has been reported, (Koivisto and Revonsuo, 2003, 2008; Ojanen et al., 2003; Pitts et al., 2012; Pitts and Martinez, 2014; see Railo et al., 2011 for a review), but only one attempt has been made thus far to compare the visual RN to the VAN (Intaite et al., 2010). Intaite et al. (2010) concluded that the visual RN and the VAN are unlikely to be the same component, although further research is necessary to verify this claim. In the auditory domain, the relationship between the aRN and ARN remains an open question.

\section{DOES THE aLPC REFLECT POST-PERCEPTUAL PROCESSING?}

The visual LPC (or simply the LP) has been associated with bistable perceptual reversals for more than 20 years and is visible in both intermittent stimulus-locked as well as continuous response-locked paradigms (Başar-Eroglu et al., 1993). Since its discovery, the LPC has been difficult to distinguish from the P300 (P3b) component, which is thought to index postperceptual updates to working memory (Donchin and Coles, 1988; Picton, 1992; McEvoy et al., 1998). Whether the task is to report perceptual reversals or to report one's percept after each trial, working memory is likely utilized for perceptual reporting purposes. Interestingly, although the onset of the visual LPC has been consistent across studies ( $2350 \mathrm{~ms})$, its duration varies considerably according to the task. For example, Pitts et al. (2008) report an LPC lasting from $\sim 350$ to beyond $700 \mathrm{~ms}$ when subjects were tasked with reporting reversals of the Necker cube, while Pitts et al. (2009) observed a shorter-duration LPC ( 350-600 ms) when the task was simply to report one's percept after each trial. Furthermore, Kornmeier and Bach (2004) manipulated the task such that subjects reported reversals on some blocks and stability on other blocks. They found a reversal-vs-stable amplitude difference in the LPC time-window regardless of task, but the overall amplitude of the LPC was larger when responses 
(vs. non-responses) to reversals were required. Overall, these findings suggest some degree of functional overlap between the LPC and the P300, in addition to similarities in polarity, timing, and scalp distribution.

If the LPC and the P300 (specifically the P3b) are identical, one might expect similar scalp topographies across visual and auditory modalities as the P3b has been found to maintain a consistent parieto-central distribution in both visual and auditory target detection tasks (Comerchero and Polich, 1999; Polich, 2007). In the current study, however, the auditory LPC was focused over more anterior scalp regions compared to the visual LPC. If the aLPC and visual LPC reflect the same underlying process, the distinct scalp distribution of the aLPC suggests an incomplete functional overlap with the P3b. To explore this issue further, we performed a correlational analysis between reversal probability and LPC amplitude, as the P3b (similar to the MMN) is known to increase in amplitude for more deviant (less probable) stimuli (Polich, 2012). Interestingly, there was a trend towards a relationship between individual subject reversal rates and aLPC amplitudes $(r=-0.39, p=0.08)$, meaning that subjects who experienced perceptual reversals less frequently tended to show larger aLPC amplitudes. While this result does not definitively link the aLPC (and LPC) with the P3b, it suggests at least a partial functional overlap due to its apparent sensitivity to percept probability.

\section{PRE-REVERSAL POSITIVITY AND THE PAIRED STIMULUS APPROACH}

When ERPs were time-locked to the first tone in the pair which was unambiguous and reversal trials were compared to stable trials, a small positivity was apparent just prior to the onset of the second (ambiguous) tone, 320-380 ms post-tone-1 (20$80 \mathrm{~ms}$ pre-tone-2). The timing of this PRP, on the cusp of a changing bistable percept, leaves open the possibility that it may reflect events that contribute causally to perceptual reversals. Prestimulus components, predictive of upcoming perceptual reversals have been reported in the past, both for the Necker cube (Britz et al., 2009; Intaite et al., 2014) as well as for binocular rivalry (Britz et al., 2011), although the functional role of such pre-reversal activity is currently unclear.

The timing of the PRP observed in the current study relates to the onset of the first tone exactly as the timing of the LPC relates to the onset of the second tone (320-380 ms posttone-onset). The topography of the PRP was also highly similar to the topography of the LPC. It is possible that the PRP and LPC represent a similar process, perhaps related to working memory. A second possibility is that pre-reversal components reflect volition or the intention to switch percepts (or attentional processes related to voluntary reversals). Subjects in the present experiment were instructed to maintain a passive approach to reversals letting them occur without voluntary control, however, it is difficult to ensure a complete lack of intentional influence over reversals in passive viewing situations. While the current study was aimed at measuring perceptual dynamics and ERPs elicited by a novel bistable auditory stimulus, future investigations will benefit from designs and analysis approaches which allow further testing in the pre-stimulus interval.

\section{CONCLUSION}

This investigation sought to expand the body of research on visual bistability into the auditory domain by pioneering a novel bistable auditory stimulus for use with the intermittent ERP paradigm. The tritone stimulus employed here successfully elicited bistable perceptions, the statistical characteristics of which were similar to those found for visual bistable figures. ERP comparisons between reversal and stable trials revealed potential auditory analogs to the previously reported RN and LPC components. These auditory components, provisionally referred to here as the aRN and aLPC, occurred slightly earlier in time and with more anterior scalp distributions compared to their visual counterparts. While the exact neuropsychological processes contributing to these ERP components remain to be specified, the current results suggest functionally-equivalent yet neuroanatomically-distinct mechanisms underlying auditory and visual bistable perception.

\section{REFERENCES}

Alpers, G. W., Ruhleder, M., Walz, N., Mühlberger, A., and Pauli, P. (2005). Binocular rivalry between emotional and neutral stimuli: a validation using fear conditioning and EEG. Int. J. Psychophysiol. 57, 25-32. doi: 10.1016/j.ijpsycho. 2005.01.008

Başar-Eroglu, C., Strüber, D., Stadler, M., Kruse, P., and Başar, E. (1993). Multistable visual perception induces a slow positive EEG wave. Int. J. Neurosci. 73, 139-151. doi: 10.3109/00207459308987220

Blake, R., O'Shea, R. P., and Mueller, T. J. (1992). Spatial zones of binocular rivalry in central and peripheral vision. Vis. Neurosci. 8, 469-478. doi: 10. $1017 / \mathrm{s} 0952523800004971$

Bool, F. H., Ernst, B., and Locher, J. (1981). MC Escher: His Life and Complete Graphic Work, with a Fully Illustrated Catalogue. New York: Abradale Press/ Harry N. Abrams.

Bregman, A. S. (1994). Auditory Scene Analysis: The Perceptual Organization of Sound. Cambridge, Massachusetts: MIT press.

Britz, J., Landis, T., and Michel, C. M. (2009). Right parietal brain activity precedes perceptual alternation of bistable stimuli. Cereb. Cortex 19, 55-65. doi: 10. 1093/cercor/bhn056

Britz, J., Pitts, M. A., and Michel, C. M. (2011). Right parietal brain activity precedes perceptual alternation during binocular rivalry. Hum. Brain Mapp. 32, 1432 1442. doi: 10.1002/hbm.21117

Chalikia, M. H., and Leinfelt, F. (2000). Listeners in Sweden perceive tritone stimuli in a manner different from that of Americans and similar to that of British listeners. J. Acoust. Soc. Am. 108, 2572. doi: 10.1121/1.4743556

Chalikia, M. H., Norberg, A. M., and Paterakis, L. (2000). Greek bilingual listeners perceive the tritone stimuli differently from speakers of english. J. Acoust. Soc. Am. 108, 2572. doi: 10.1121/1.4743555

Clark, V. P., Fan, S., and Hillyard, S. A. (1994). Identification of early visual evoked potential generators by retinotopic and topographic analyses. Hum. Brain Mapp. 2, 170-187. doi: 10.1002/hbm.460020306

Comerchero, M. D., and Polich, J. (1999). P3a and P3b from typical auditory and visual stimuli. Clin. Neurophysiol. 110, 24-30. doi: 10.1016/s01685597(98)00033-1

Cusack, R. (2005). The intraparietal sulcus and perceptual organization. J. Cogn. Neurosci. 17, 641-651. doi: 10.1162/0898929053467541

Davis, P. A. (1939). Effects of acoustic stimuli on the waking human brain. $J$. Neurophysiol. 2, 494-499.

Deutsch, D. (1986). A musical paradox. Music Percept. 3, 275-280. doi: 10. 2307/40285337

Deutsch, D. (1987). The tritone paradox: effects of spectral variables. Percept. Psychophys. 41, 563-575. doi: 10.3758/bf03210490

Deutsch, D. (1991). The tritone paradox: an influence of language on music perception. Music Percept. 8, 335-347. doi: 10.2307/40285517

Deutsch, D. (1992). Some new pitch paradoxes and their implications. Philos. Trans. R. Soc. Lond. B Biol. Sci. 336, 391-397. doi: 10.1098/rstb.1992.0073

Deutsch, D. (1994). The tritone paradox: some further geographical correlates. Music Percept. 12, 125-136. doi: 10.2307/40285758 
Deutsch, D. (1997). The tritone paradox: a link between music and speech. Curr. Dir. Psychol. Sci. 6, 174-180. doi: 10.1111/1467-8721.ep10772951

Deutsch, D., Kuyper, W. L., and Fisher, Y. (1987). The tritone paradox: its presence and form of distribution in a general population. Music Percept. 5, 79-92. doi: $10.2307 / 40285386$

Donchin, E., and Coles, M. G. (1988). Is the P300 component a manifestation of context updating? Behav. Brain Sci. 11, 357-374. doi: 10. 1017/s0140525x00058027

Dykstra, A. R., Halgren, E., Thesen, T., Carlson, C. E., Doyle, W., Madsen, J. R., et al. (2011). Widespread brain areas engaged during a classical auditory streaming task revealed by intracranial EEG. Front. Hum. Neurosci. 5:74. doi: 10. 3389/fnhum.2011.00074

Ehm, W., Bach, M., and Kornmeier, J. (2011). Ambiguous figures and binding: EEG frequency modulations during multistable perception. Psychophysiology 48, 547-558. doi: 10.1111/j.1469-8986.2010.01087.x

Gamble, M. L., and Luck, S. J. (2011). N2ac: an ERP component associated with the focusing of attention within an auditory scene. Psychophysiology 48, 1057-1068. doi: 10.1111/j.1469-8986.2010.01172.x

Genç, E., Bergmann, J., Singer, W., and Kohler, A. (2011). Interhemispheric connections shape subjective experience of bistable motion. Curr. Biol. 21, 1494 1499. doi: 10.1016/j.cub.2011.08.003

Giangrande, J. (1998). The tritone paradox: effects of pitch class and position of the spectral envelope. Music Percept. 15, 253-264. doi: 10.2307/40285767

Giangrande, J., Tuller, B., and Kelso, J. A. S. (2003). Perceptual dynamics of circular pitch. Music Percept. 20, 241-262. doi: 10.1525/mp.2003.20.3.241

Gutschalk, A., and Dykstra, A. (2014). Functional imaging of auditory scene analysis. Hear. Res. 307, 98-110. doi: 10.1016/j.heares.2013.08.003

Gutschalk, A., Micheyl, C., and Oxenham, A. J. (2008). Neural correlates of auditory perceptual awareness under informational masking. PLoS Biol. 6:e138. doi: 10 . 1371/journal.pbio.0060138

Gutschalk, A., Micheyl, C., Melcher, J. R., Rupp, A., Scherg, M., and Oxenham, A. J. (2005). Neuromagnetic correlates of streaming in human auditory cortex. J. Neurosci. 25, 5382-5388. doi: 10.1523/jneurosci.0347-05.2005

Hickey, C., McDonald, J. J., and Theeuwes, J. (2006). Electrophysiological evidence of the capture of visual attention. J. Cogn. Neurosci. 18, 604-613. doi: 10. 1162/jocn.2006.18.4.604

Hillyard, S. A., Hink, R. F., Schwent, V. L., and Picton, T. W. (1973). Electrical signs of selective attention in the human brain. Science 182, 177-180. doi: 10 . 1126/science.182.4108.177

Intaite, M., Koivisto, M., and Castelo-Branco, M. (2014). Event-related potential responses to perceptual reversals are modulated by working memory load. Neuropsychologia 56, 428-438. doi: 10.1016/j.neuropsychologia.2014.02.016

Intaite, M., Koivisto, M., and Revonsuo, A. (2013). Perceptual reversals of Necker stimuli during intermittent presentation with limited attentional resources. Psychophysiology 50, 82-96. doi: 10.1111/j.1469-8986.2012.01486.x

Intaitė, M., Koivisto, M., Rukšėnas, O., and Revonsuo, A. (2010). Reversal negativity and bistable stimuli: attention, awareness, or something else? Brain Cogn. 74, 24-34. doi: 10.1016/j.bandc.2010.06.002

Kaneoke, Y., Urakawa, T., Hirai, M., Kakigi, R., and Murakami, I. (2009). Neural basis of stable perception of an ambiguous apparent motion stimulus. Neuroscience 159, 150-160. doi: 10.1016/j.neuroscience.2008.12.014

Kashino, M., and Kondo, H. M. (2012). Functional brain networks underlying perceptual switching: auditory streaming and verbal transformations. Philos. Trans. R. Soc. Lond. B Biol. Sci. 367, 977-987. doi: 10.1098/rstb.2011.0370

Kim, Y. J., Grabowecky, M., Suzuki, S., and Rubin, N. (2009). Bistability of flicker vs. rotational apparent motion: Psychophysics and Steady-State Visual Evoked Potentials (SSVEP). J. Vis. 9: 675. doi: 10.1167/9.8.675

Koivisto, M., and Revonsuo, A. (2003). An ERP study of change detection, change blindness and visual awareness. Psychophysiology 40, 423-429. doi: 10. $1111 / 1469-8986.00044$

Koivisto, M., and Revonsuo, A. (2008). Comparison of event-related potentials in attentional blink and repetition blindness. Brain Res. 1189, 115-126. doi: 10. 1016/j.brainres.2007.10.082

Koivisto, M., and Revonsuo, A. (2010). Event-related brain potential correlates of visual awareness. Neurosci. Biobehav. Rev. 34, 922-934. doi: 10.1016/j.neubiorev. 2009.12.002

Kondo, H. M., and Kashino, M. (2007). Neural mechanisms of auditory awareness underlying verbal transformations. Neuroimage 36, 123-130. doi: 10.1016/j. neuroimage.2007.02.024
Kondo, H. M., and Kashino, M. (2009). Involvement of the thalamocortical loop in the spontaneous switching of percepts in auditory streaming. J. Neurosci. 29, 12695-12701. doi: 10.1523/JNEUROSCI.1549-09.2009

Kondo, H. M., Kitagawa, N., Kitamura, M. S., Koizumi, A., Nomura, M., and Kashino, M. (2012). Separability and commonality of auditory and visual bistable perception. Cereb. Cortex 22, 1915-1922. doi: 10.1093/cercor/ bhr266

Königs, L., and Gutschalk, A. (2012). Functional lateralization in auditory cortex under informational masking and in silence. Eur. J. Neurosci. 36, 3283-3290. doi: 10.1111/j.1460-9568.2012.08240.x

Kornmeier, J., and Bach, M. (2004). Early neural activity in Necker-cube reversal: evidence for low-level processing of a gestalt phenomenon. Psychophysiology 41, 1-8. doi: 10.1046/j.1469-8986.2003.00126.x

Kornmeier, J., and Bach, M. (2005). The Necker cube-an ambiguous figure disambiguated in early visual processing. Vision Res. 45, 955-960. doi: 10.1016/j. visres.2004.10.006

Kornmeier, J., and Bach, M. (2006). Bistable perception-along the processing chain from ambiguous visual input to a stable percept. Int. J. Psychophysiol. 62, 345-349. doi: 10.1016/j.ijpsycho.2006.04.007

Kornmeier, J., and Bach, M. (2009). Object perception: when our brain is impressed but we do not notice it. J. Vis. 9:7. doi: 10.1167/9.1.7

Kornmeier, J., and Bach, M. (2012). Ambiguous figures-what happens in the brain when perception changes but not the stimulus. Front. Hum. Neurosci. 6:51. doi: 10.3389/fnhum.2012.00051

Kornmeier, J., Ehm, W., Bigalke, H., and Bach, M. (2007). Discontinuous presentation of ambiguous figures: how interstimulus-interval durations affect reversal dynamics and ERPs. Psychophysiology 44, 552-560. doi: 10.1111/j.1469-8986. 2007.00525.x

Kornmeier, J., Hein, C. M., and Bach, M. (2009). Multistable perception: when bottom-up and top-down coincide. Brain Cogn. 69, 138-147. doi: 10.1016/j. bandc.2008.06.005

Kornmeier, J., Wörner, R., Riedel, A., Bach, M., and van Elst, L. T. (2014). A different view on the checkerboard? Alterations in early and late visually evoked EEG potentials in asperger observers. PLoS One 9:e90993. doi: 10.1371/journal. pone.0090993

Lansing, R. W. (1964). Electroencephalographic correlates of binocular rivalry in man. Science 146, 1325-1327. doi: 10.1126/science.146.3649.1325

Lemmo, G. (2006). Cheetahs [Photograph]. Photograph appeared in National Geographic World Magazine, October 1997; Photograph taken in Masai Mara Game Reserve, Kenya, 1993. Available online at: http://www.gerrylemmo.com/gallery. asp?ID=193.

Leopold, D. A., and Logothetis, N. K. (1999). Multistable phenomena: changing views in perception. Trends Cogn. Sci. 3, 254-264. doi: 10.1016/s13646613(99)01332-7

Leopold, D. A., Wilke, M., Maier, A., and Logothetis, N. K. (2002). Stable perception of visually ambiguous patterns. Nat. Neurosci. 5, 605-609. doi: 10 1038/nn0602-851

Long, G. M., and Toppino, T. C. (2004). Enduring interest in perceptual ambiguity: alternating views of reversible figures. Psychol. Bull. 130, 748-768. doi: 10. 1037/0033-2909.130.5.748

Luck, S. J. (2012). "Electrophysiological correlates of the focusing of attention within complex visual scenes: N2pc and related ERP components," in The Oxford Handbook of Event-Related Potential Components, eds S. J. Luck and E. S. Kappenman (New York: Oxford university press), 329-360.

Luck, S. J., and Hillyard, S. A. (1994). Spatial filtering during visual search: evidence from human electrophysiology. J. Exp. Psychol. Hum. Percept. Perform. 20, 1000 1014. doi: 10.1037//0096-1523.20.5.1000

McEvoy, L. K., Smith, M. E., and Gevins, A. (1998). Dynamic cortical networks of verbal and spatial working memory: effects of memory load and task practice. Cereb. Cortex 8, 563-574. doi: 10.1093/cercor/8.7.563

Meng, M., and Tong, F. (2004). Can attention selectively bias bistable perception? Differences between binocular rivalry and ambiguous figures. J. Vis. 4, 539-551. doi: 10.1167/4.7.2

Müller, T. J., Federspiel, A., Fallgatter, A. J., and Strik, W. K. (1999). EEG signs of vigilance fluctuations preceding perceptual flips in multistable illusionary motion. Neuroreport 10, 3423-3427. doi: 10.1097/00001756-199911080-00030

Näätänen, R., Gaillard, A. W., and Mäntysalo, S. (1978). Early selective-attention effect on evoked potential reinterpreted. Acta Psychol. (Amst) 42, 313-329. doi: 10.1016/0001-6918(78)90006-9 
Näätänen, R., and Kreegipuu, K. (2012). The Mismatch Negativity (MMN). Oxford: Oxford University Press.

Näätänen, R., Pakarinen, S., Rinne, T., and Takegata, R. (2004). The mismatch negativity (MMN): towards the optimal paradigm. Clin. Neurophysiol. 115, 140144. doi: 10.1016/j.clinph.2003.04.001

Näätänen, R., and Picton, T. (1987). The N1 wave of the human electric and magnetic response to sound: a review and an analysis of the component structure. Psychophysiology 24, 375-425. doi: 10.1111/j.1469-8986.1987.tb00311.x

Necker, L. A. (1832). LXI. Observations on some remarkable optical phaenomena seen in Switzerland; and on an optical phaenomenon which occurs on viewing a figure of a crystal or geometrical solid. Philos. Mag. Ser. 3 1, 329-337. doi: 10. 1080/14786443208647909

Ojanen, V., Revonsuo, A., and Sams, M. (2003). Visual awareness of low-contrast stimuli is reflected in event-related brain potentials. Psychophysiology 40, 192197. doi: 10.1111/1469-8986.00021

Orbach, J., Ehrlich, D., and Heath, H. A. (1963). Reversibility of the necker cube: an examination of the concept of "satiation of orientation". Percept. Mot. Skills 17, 439-458. doi: 10.2466/pms.1963.17.2.439

Orbach, J., Zucker, E., and Olson, R. (1966). Reversibility of the Necker cube: VII. Reversal rate as a function of figure-on and figure-off durations. Percept. Mot. Skills 22, 615-618. doi: 10.2466/pms.1966.22.2.615

Picton, T. W. (1992). The P300 wave of the human event-related potential. J. Clin. Neurophysiol. 9, 456-479. doi: 10.1097/00004691-199210000-00002

Pitts, M. A., and Britz, J. (2011). Insights from intermittent binocular rivalry and EEG. Front. Hum. Neurosci. 5:107. doi: 10.3389/fnhum.2011.00107

Pitts, M. A., and Martinez, A. (2014). "Contour integration: sensory, perceptual and attention-based ERP components," in Cognitive Electrophysiology of Attention: Signals of the Mind, ed G. R. Mangun (San Diego, CA: Academic Press), 178-189.

Pitts, M. A., Gavin, W. J., and Nerger, J. L. (2008). Early top-down influences on bistable perception revealed by event-related potentials. Brain Cogn. 67, 11-24. doi: 10.1016/j.bandc.2007.10.004

Pitts, M. A., Martínez, A., and Hillyard, S. A. (2012). Visual processing of contour patterns under conditions of inattentional blindness. J. Cogn. Neurosci. 24, 287303. doi: 10.1162/jocn_a_00111

Pitts, M. A., Martínez, A., Stalmaster, C., Nerger, J. L., and Hillyard, S. A. (2009). Neural generators of ERPs linked with Necker cube reversals. Psychophysiology 46, 694-702. doi: 10.1111/j.1469-8986.2009.00822.x

Pitts, M. A., Nerger, J. L., and Davis, T. J. (2007). Electrophysiological correlates of perceptual reversals for three different types of multistable images. J. Vis. 7:6. doi: $10.1167 / 7.1 .6$

Polich, J. (2007). Updating P300: an integrative theory of P3a and P3b. Clin. Neurophysiol. 118, 2128-2148. doi: 10.1016/j.clinph.2007.04.019

Polich, J. (2012). “Neuropsychology of P300,” in Oxford Handbook of Event-Related Potential Components, eds S. J. Luck and E. S. Kappenman (New York: Oxford University Press), 159-188.

Pressnitzer, D., and Hupé, J. M. (2006). Temporal dynamics of auditory and visual bistability reveal common principles of perceptual organization. Curr. Biol. 16, 1351-1357. doi: 10.1016/j.cub.2006.05.054

Ragozzine, F., and Deutsch, D. (1994). A regional difference in perception of the tritone paradox within the United States. Music Percept. 12, 213-225. doi: 10. 2307/40285652

Railo, H., Koivisto, M., and Revonsuo, A. (2011). Tracking the processes behind conscious perception: a review of event-related potential correlates of visual consciousness. Conscious. Cogn. 20, 972-983. doi: 10.1016/j.concog.2011.03.019

Repp, B. H. (1994). The tritone paradox and the pitch range of the speaking voice: a dubious connection. Music Percept. 12, 227-255. doi: 10.2307/ 40285653

Repp, B. H. (1997). Spectral envelope and context effects in the tritone paradox. Perception 26, 645-666. doi: 10.1068/p260645

Repp, B. H., and Knoblich, G. (2007). Action can affect auditory perception. Psychol. Sci. 18, 6-7. doi: 10.1111/j.1467-9280.2007.01839.x

Repp, B. H., and Thompson, J. M. (2010). Context sensitivity and invariance in perception of octave-ambiguous tones. Psychol. Res. 74, 437-456. doi: 10. 1007/s00426-009-0264-9
Robitaille, N., and Jolicoeur, P. (2006). Fundamental properties of the N2pc as an index of spatial attention: effects of masking. Can. J. Exp. Psychol. 60, 79-89. doi: $10.1037 /$ cjep2006011

Rubin, E. (1958). "Figure and ground," in Readings in Perception, ed D. Beardslee (Princeton, N. J.: Van Nostrand), 194-203.

Sato, M., Baciu, M., Loevenbruck, H., Schwartz, J. L., Cathiard, M. A., Segebarth, C., et al. (2004). Multistable representation of speech forms: a functional MRI study of verbal transformations. Neuroimage 23, 1143-1151. doi: 10.1016/j. neuroimage.2004.07.055

Schadwinkel, S., and Gutschalk, A. (2011). Transient bold activity locked to perceptual reversals of auditory streaming in human auditory cortex and inferior colliculus. J. Neurophysiol. 105, 1977-1983. doi: 10.1152/jn.004 61.2010

Shepard, R. N. (1964). Circularity in judgments of relative pitch. J. Acoust. Soc. Am. 36:2346. doi: 10.1121/1.1919362

Shepard, R. N. (1982). Geometrical approximations to the structure of musical pitch. Psychol. Rev. 89, 305-333. doi: 10.1037//0033-295x.89.4.305

Snyder, J. S., Alain, C., and Picton, T. W. (2006). Effects of attention on neuroelectric correlates of auditory stream segregation. J. Cogn. Neurosci. 18, 1-13. doi: 10. $1162 / 089892906775250021$

Snyder, J. S., Gregg, M. K., Weintraub, D. M., and Alain, C. (2012). Attention, awareness and the perception of auditory scenes. Front. Psychol. 3:15. doi: 10. 3389/fpsyg.2012.00015

Snyder, J. S., Holder, W. T., Weintraub, D. M., Carter, O. L., and Alain, C. (2009). Effects of prior stimulus and prior perception on neural correlates of auditory stream segregation. Psychophysiology 46, 1208-1215. doi: 10.1111/j.1469-8986. 2009.00870.x

Spehlmann, R. (1965). The averaged electrical responses to diffuse and to patterned light in the human. Electroencephalogr. Clin. Neurophysiol. 19, 560-569. doi: 10. 1016/0013-4694(65)90241-5

Sperling, G., van Santen, J. P., and Burt, P. J. (1985). Three theories of stroboscopic motion detection. Spat. Vis. 1, 47-56. doi: 10.1163/156856885x00071

Sterzer, P., Kleinschmidt, A., and Rees, G. (2009). The neural bases of multistable perception. Trends Cogn. Sci. 13, 310-318. doi: 10.1016/j.tics.2009. 04.006

Strüber, D., Rach, S., Trautmann-Lengsfeld, S. A., Engel, A. K., and Herrmann, C. S. (2014). Antiphasic $40 \mathrm{~Hz}$ oscillatory current stimulation affects bistable motion perception. Brain Topogr. 27, 158-171. doi: 10.1007/s10548-013-0294-x

Tong, F., Meng, M., and Blake, R. (2006). Neural bases of binocular rivalry. Trends Cogn. Sci. 10, 502-511. doi: 10.1016/j.tics.2006.09.003

Tong, F., Nakayama, K., Vaughan, J. T., and Kanwisher, N. (1998). Binocular rivalry and visual awareness in human extrastriate cortex. Neuron 21, 753-759. doi: 10. 1016/s0896-6273(00)80592-9

Tsuchiya, N., and van Boxtel, J. (2013). Introduction to research topic: attention and consciousness in different senses. Front. Psychol. 4:249. doi: 10.3389/fpsyg. 2013.00249

Wiegand, K., and Gutschalk, A. (2012). Correlates of perceptual awareness in human primary auditory cortex revealed by an informational masking experiment. Neuroimage 61, 62-69. doi: 10.1016/j.neuroimage.2012.02.067

Conflict of Interest Statement: The authors declare that the research was conducted in the absence of any commercial or financial relationships that could be construed as a potential conflict of interest.

Received: 17 May 2014; accepted: 14 July 2014; published online: 08 August 2014. Citation: Davidson GD and Pitts MA (2014) Auditory event-related potentials associated with perceptual reversals of bistable pitch motion. Front. Hum. Neurosci. 8:572. doi: 10.3389/fnhum.2014.00572

This article was submitted to the journal Frontiers in Human Neuroscience. Copyright (C) 2014 Davidson and Pitts. This is an open-access article distributed under the terms of the Creative Commons Attribution License (CC BY). The use, distribution or reproduction in other forums is permitted, provided the original author (s) or licensor are credited and that the original publication in this journal is cited, in accordance with accepted academic practice. No use, distribution or reproduction is permitted which does not comply with these terms. 